\title{
Disponibilidad de datos y perfil de morbilidad en Colombia
}

\author{
Oscar Bernal, ${ }^{1}$ Juan Camilo Forero, ${ }^{1}$ María del Pilar Villamil ${ }^{2}$ \\ y Rafael Pino ${ }^{2}$
}

Forma de citar

Bernal O, Forero JC, Villamil MP, Pino R. Disponibilidad de datos y perfil de morbilidad en Colombia. Rev Panam Salud Publica. 2012;31(3):181-7.

RESUMEN Objetivo. Caracterizar la morbilidad de la población colombiana según causas diagnósticas, género, edad, región y régimen de cobertura en salud, evaluando al mismo tiempo la disponibilidad y la calidad de la información sobre enfermedades en el país. Estudio de tipo descriptivo, transversal, que analiza la morbilidad atendida en consulta externa, hospitalización y urgencias en Colombia durante el quinquenio 2004-2008.

Métodos. Se utilizaron los "Registros individuales de prestación de servicios" (RIPS) de Colombia, clasificándose los diagnósticos según los grupos de causas definidos por la Organización Mundial de la Salud. Cada grupo de causas para utilización de servicios se discriminó por región geográfica, género y régimen de afiliación al sistema de salud.

Resultados. Los diagnósticos de enfermedades transmisibles se encontraron más concentrados en los grupos de edad inferiores, mientras que las no transmisibles fueron más frecuentes en los grupos etarios superiores. Las externalidades (accidentes, lesiones autoinfligidas y violencia) fueron una causa importante de morbilidad y afectaron con más fuerza a la población de entre 5 y 44 años de edad. Las patologías transmisibles fueron más prevalentes en mujeres $(39,98 \%)$ que en hombres $(28 \%)$, en tanto que los hombres se vieron más afectados por las externalidades que las mujeres (18,5 y 7,3\%, respectivamente).

Conclusiones. Si bien en Colombia han aumentado los diagnósticos de enfermedades no transmisibles, las infecciones siguen teniendo una presencia relevante en todos los grupos de edad y escenarios de prestación de servicios de salud. Tal situación exige revisar las politicas sanitarias para orientarlas no solo a mejorar la salud de la población sino también a reducir la brecha de servicios que existe entre las diferentes regiones del país. Por otro lado, aun cuando los RIPS constituyen una valiosa fuente de datos sanitarios, es preciso fortalecerlos con el propósito de lograr una cobertura de información que abarque a toda o por lo menos a la gran mayoría de la población colombiana.

Palabras clave Morbilidad; sistemas de información; registros de enfermedades; registros médicos; Colombia.

La planificación y la formulación de políticas públicas en salud dependen en gran medida de la disponibilidad de información efectiva y oportuna (1). Más

\footnotetext{
1 Universidad de los Andes, Facultad de Medicina, Área de Salud Pública, Bogotá, Colombia. La correspondencia se debe dirigir a Oscar Bernal. Correo electrónico: obernal@uniandes.edu.co

2 Universidad de los Andes, Facultad de Ingeniería, Departamento de Ingeniería de Sistemas y Computación, Bogotá, Colombia.
}

aún, es bien reconocida la importancia clave que tienen la generación, el flujo y el análisis de esta información para asegurar que las decisiones tomadas sean confiables, factibles y articuladas con la realidad a la que van a ser dirigidas (2).

Colombia cuenta con diferentes fuentes de datos provenientes del Sistema de Protección Social, el cual tiene bases que incluyen, entre otros, datos sobre seguros, prestación de servicios, empleo y pensiones (3). Una de estas bases de datos son los "Registros individuales de prestación de servicios" (RIPS), donde se consignan los servicios de salud prestados en el país para cada año, detallados según sean procedimientos, consulta externa, hospitalización y urgencias. Aun cuando algunos autores han cuestionado la idoneidad de los RIPS, dada la (abun- 
dante) cantidad y la calidad —observada por los autores- de los datos que almacenan representan un valiosa fuente de información sanitaria (4). Dicho esto, el país no cuenta con un perfil de morbilidad nacional claramente establecido.

El objetivo del presente estudio fue justamente caracterizar la morbilidad de la población colombiana según causas diagnósticas, género, edad, región y régimen de cobertura en salud, evaluando al mismo tiempo la disponibilidad y la calidad de la información sobre morbilidad en el país.

\section{MATERIALES Y MÉTODOS}

Se realizó un estudio descriptivo, transversal, acerca de los diagnósticos más frecuentes en consulta, hospitalización y urgencias en Colombia durante el período 2004 a 2008. La información -consignada en los RIPS para 2001-2008 - fue proporcionada a la Universidad de los Andes por el Ministerio de Protección Social. Dado que en un primer análisis se encontró que la calidad de los datos para 2001-2003 era inferior a la de los siguientes 5 años, los resultados aquí expuestos corresponden únicamente al análisis de los años 2004 a 2008. Asimismo, no se tuvieron en cuenta los datos sobre procedimientos porque se consideró que tal información se deriva de los diagnósticos realizados en los otros tres escenarios de prestación de servicios. La unidad de análisis fue el diagnóstico.

Utilizando el programa de análisis estadístico STATA versión 11, se hizo una validación interna de los datos para reclasificarlos según su grado de solidez (p. ej. elementos faltantes en la información que presentó errores). No se tuvieron en cuenta los diagnósticos clasificados como "confirmados repetidos" en las bases de datos.

Se revisaron asimismo los informes de las Empresas Administradoras de Planes de Beneficios (EAPB), ${ }^{3}$ clasificándolos conforme a la EAPB que los publicó, con objeto de determinar la cobertura informativa anual y estimar los porcentajes de población afiliada a estas entidades. Adicionalmente, se incorporaron al análisis las series de población reportadas por el Departamento Administrativo Nacional

\footnotetext{
3 Las EAPB incluyen entidades promotoras de salud del régimen contributivo y subsidiado, empresas solidarias de salud, asociaciones mutuales, cajas de compensación familiar y entidades que administran planes adicionales de salud.
}

de Estadística de Colombia, las cuales permitieron establecer tasas de utilización de servicios discriminadas por género, edad y escenario de consulta (5). A continuación, las etiologías de los diagnósticos fueron organizadas según grupos de edad y género, y se establecieron grupos diagnósticos según fueran "infecciosos, perinatales, maternos y nutricionales" (grupo 1), "no transmisibles" (grupo 2) y [provocados por] "externalidades" tales como accidentes, violencia y lesiones auto-infligidas (grupo 3). Esta reagrupación metodológica concuerda con la propuesta por la Organización Mundial de la Salud (OMS) en un estudio sobre carga de enfermedad publicado en 2004 y utilizado en otros trabajos de investigación $(6,7)$. Para organizar la información, se calculó el promedio de causas de morbilidad correspondiente a cada escenario de atención y a cada período estudiado. También se analizó la demanda de servicios por régimen de afiliación al sistema de salud (contributivo, subsidiado y "no asegurados"), calculándose la población absoluta dentro de cada régimen a partir de los porcentajes de afiliación y las series de población del país para cada año (5, 8-10). Finalmente, se creó un indicador de razón entre enfermedades transmisibles y no transmisibles - dividiendo la cantidad de registros del grupo 1 entre los del grupo 2- que permitió determinar cuál de los dos grupos es más frecuente en cada departamento del país y su comportamiento en el tiempo.

CUADRO 1. Población (número) afiliada al sistema de salud colombiano, según género, edad y tipo de usuario, 2004-2008

\begin{tabular}{|c|c|c|c|c|c|}
\hline Población & 2004 & 2005 & 2006 & 2007 & 2008 \\
\hline Total registrada & 15051564 & 17881969 & 19545176 & 17724488 & 8563309 \\
\hline \multicolumn{6}{|l|}{ Género } \\
\hline Femenino & 9472738 & 11340136 & 12422105 & 11406512 & 5485599 \\
\hline Masculino & 5578826 & 6541833 & 7123071 & 6317976 & 3077710 \\
\hline \multicolumn{6}{|l|}{ Edad } \\
\hline $0-4$ & 2080111 & 2397989 & 2667355 & 2274332 & 1280569 \\
\hline $05-14$ & 1934955 & 2171864 & 2368530 & 1876664 & 821026 \\
\hline $15-44$ & 7257100 & 8551335 & 9011931 & 8358197 & 3883340 \\
\hline $45-59$ & 2043830 & 2547037 & 2801392 & 2708070 & 1327203 \\
\hline$>60$ & 1735568 & 2213744 & 2695968 & 2507225 & 1251171 \\
\hline \multicolumn{6}{|l|}{ Tipo de usuario } \\
\hline Contributivo & 7482247 & 7386967 & 5129964 & 5490174 & 2763199 \\
\hline Subsidiado & 4314424 & 5352593 & 7328863 & 8023196 & 3078917 \\
\hline No asegurado & 2844892 & 3955034 & 5133408 & 2854522 & 1734543 \\
\hline Particular & 181996 & 537191 & 834258 & 547429 & 509017 \\
\hline Otro & 225106 & 578213 & 962930 & 641806 & 345176 \\
\hline Faltantes ${ }^{a}$ & 2899 & 71971 & 155753 & 167361 & 132457 \\
\hline
\end{tabular}

a Faltantes corresponde a registros que no cumplen con los valores establecidos para cada variable y fueron reclasificados.

\section{RESULTADOS}

En cuanto a la cobertura y disponibilidad de datos, se halló que en 2005 un promedio de $45 \%$ de las administradoras de salud generaron informes de morbilidad alguna vez durante el año, en tanto que $26 \%$ lo hicieron mensual y consistentemente durante los 12 meses. En 2008, $33 \%$ reportaron por lo menos una vez y $6 \%$ lo hicieron mensualmente todo el año. De las 136 administradoras registradas, 62 reportaron en 2005 y 47 en 2008. Se encontraron disponibles informes de administradoras que agrupan alrededor de $55 \%$ de los afiliados al régimen contributivo y $47 \%$ al subsidiado. Las variables analizadas no superaron para ningún año o escenario un porcentaje de error superior al 1,5\% de los registros. El tipo de afiliación al sistema de salud colombiano fue la variable que más errores presentó en todos los años y escenarios.

Se constató la existencia de un promedio de 15753301 registros para el período 2004-2008 (cuadro 1), con un promedio de 13800000 en consulta externa, 1112000 en urgencias y 834300 en hospitalizaciones. La proporción de mujeres que recibieron servicios fue cercana al doble que la de los hombres, con $63,6 \%$ y $36,3 \%$, respectivamente.

Si bien el grupo de edad con más eventos registrados fue el de 15 a 44 años, al ajustar por población se observó que los grupos de edad que más demandaron servicios fueron el de 0 a 4 años y el de 
mayores de 60 años, con tasas de $49 \%$ y $53 \%$ respectivamente. En los menores de 5 años se halló un predominio de patologías transmisibles ( $66 \%$ de los eventos), mientras que el grupo de 5 a 14 años registró una proporción mayoritaria de patologías no transmisibles $(45 \%)$ y un porcentaje casi tres veces más alto de externalidades que los menores de 5 años de edad con $18 \%$ y $7 \%$ respectivamente (figura 1). En el grupo de 15 a 44 años las enfermedades transmisibles fueron levemente más frecuentes que en el grupo de 5 a 14 años (39\% vs. 37\%, respectivamente), mientras que algo similar ocurrió con las enfermedades no transmisibles ( $48 \%$ y $45 \%$, respectivamente). Las externalidades representaron $13 \%$ del total de diagnósticos.

En la figura 1 se puede observar que, dentro del grupo etario de 45 a 59 años, las patologías no transmisibles fueron con mucho las más frecuentes (74\%), lejos del 14\% que correspondió a las enfermedades transmisibles y del 13\% que registraron las externalidades. Asimismo, se reportaron más enfermedades transmisibles en mujeres (40\%) que en hombres (28\%), en tanto que lo opuesto ocurrió con las externalidades ( $7 \%$ y $19 \%$, respectivamente) (figura 2). El grupo etario que mostró más diferencia por género fue el de 15 a 44 años, particularmente en externalidades y patologías transmisibles. Por otro lado, en el caso de consulta la razón enfermedades transmisibles/no transmisibles no registró variaciones importantes a lo largo del período estudiado, algo que sí se dio en hospitalización, donde las transmisibles fueron bastante más frecuentes al inicio que al final del período de análisis (figura 3). Las urgencias mostraron un comportamiento contrario, con una razón para las enfermedades transmisibles que pasó de 0,9 en 2004 a 1,0 en 2008.

Al contrastar las razones enfermedades transmisibles/no transmisibles correspondientes a cada departamento del país, se encontró que en promedio las no transmisibles fueron más frecuentes que las transmisibles en todas las regiones. Sin embargo, cuando se observó el cambio del indicador promedio en los 5 años del análisis, se halló que en los departamentos Atlántico, Bolívar, Huila, Nariño, Norte de Santander y Putumayo, entre otros, hubo un aumento de las patologías transmisibles, mientras que en Cauca, Santander y Tolima se dio una reducción de las patologías trans-

FIGURA 1. Morbilidad por enfermedades transmisibles, no transmisibles y externalidades, por grupo de edad (porcentajes redondeados), Colombia, 2004-2008

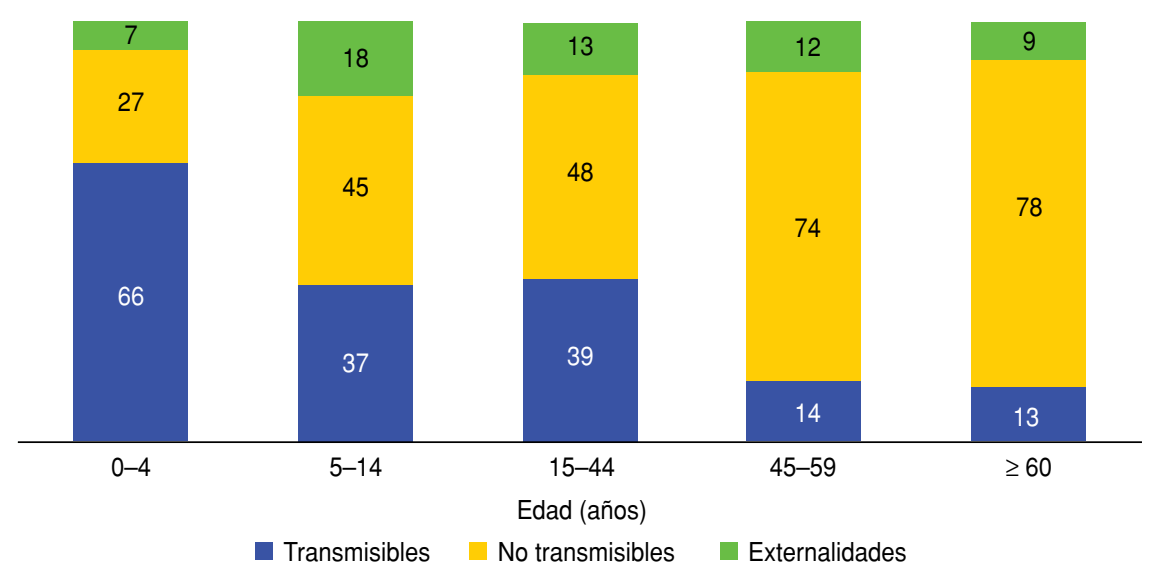

Fuente: elaboración de los autores.

FIGURA 2. Morbilidad por enfermedades transmisibles, no transmisibles y externalidades, según género (porcentajes redondeados), Colombia, 2004-2008

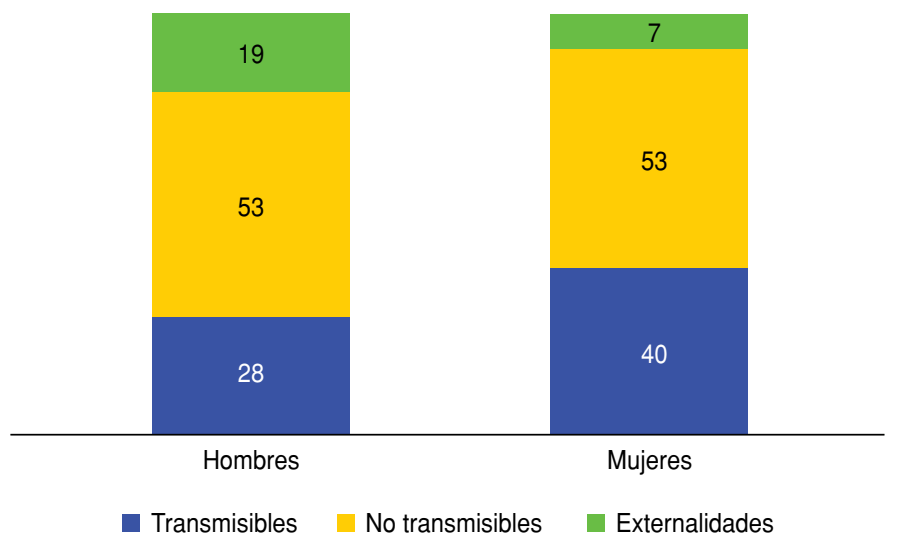

Fuente: elaboración de los autores.

FIGURA 3. Razón enfermedades transmisibles/no transmisibles, según casos registrados en consulta, urgencias u hospitalización, Colombia, 2004-2008

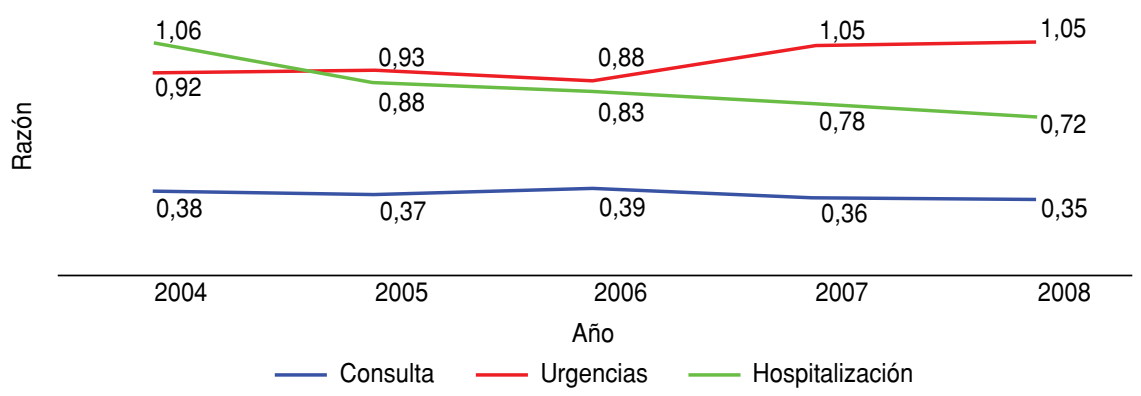

Fuente: elaboración de los autores.

misibles frente a las no transmisibles (figura 4).

En cuanto a los tipos de afiliación de cobertura en salud, en 2004 la proporción de servicios prestados se concentró más en el régimen contributivo con un $49,7 \%$, seguido por el subsidiado con $28,6 \%$ de los servicios y por la población no asegurada con $18,9 \%$, mientras que en 2006 el régimen subsidiado superó 
FIGURA 4. Variaciones en la razón enfermedades transmisibles/no transmisibles durante el período 2004-2008, por departamentos, Colombia

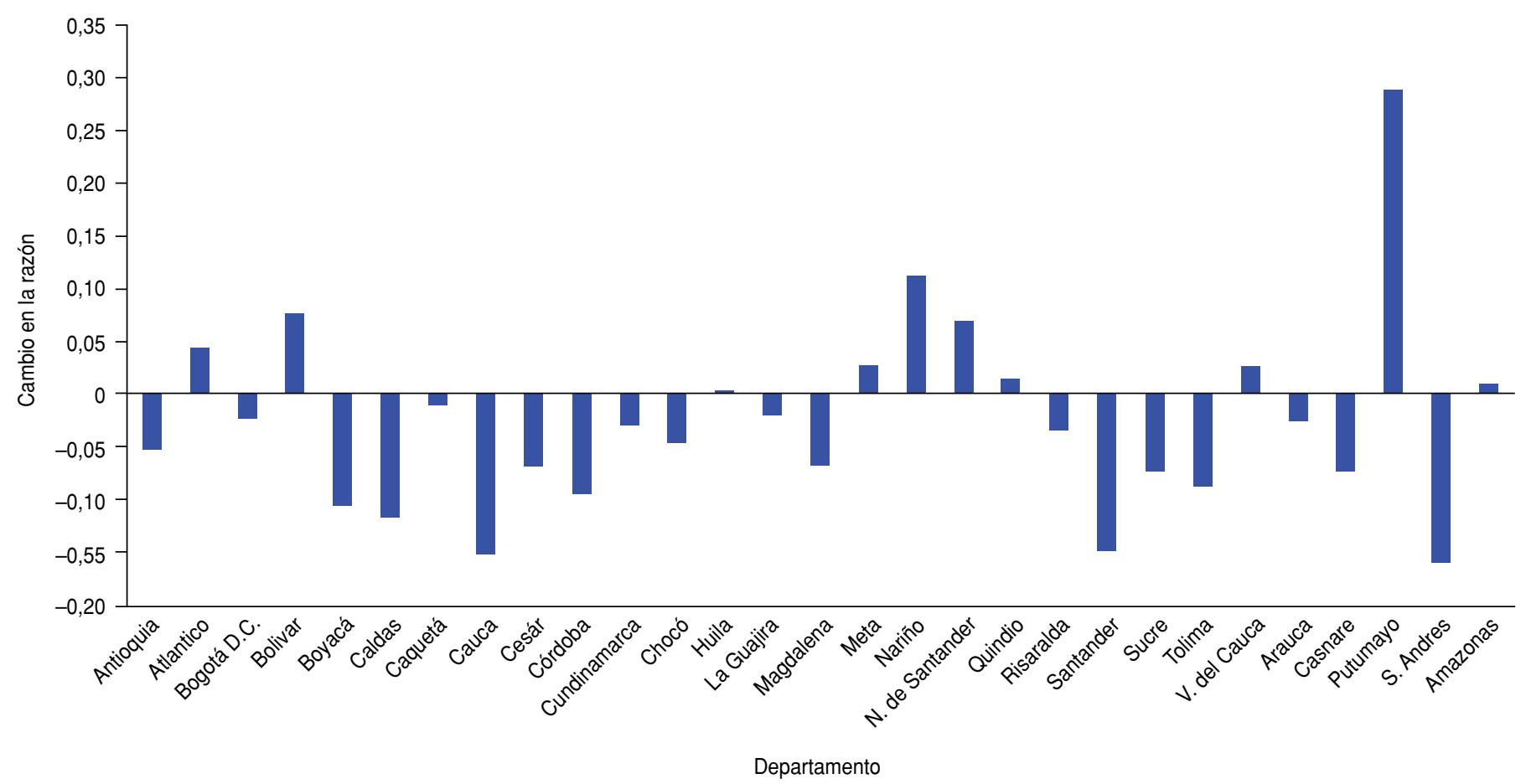

Fuente: elaboración de los autores.

al contributivo, con una proporción de servicios de $37,5 \%$ frente a $26,2 \%$, respectivamente, y en 2007 esa brecha se amplió todavía más, con participaciones de $45,3 \%$ y $31 \%$ respectivamente - durante este último año la población no asegurada recibió $16,1 \%$ de los servicios registrados. En 2008, el régimen subsidiado registró 35,9\% de los servicios y el contributivo $32,2 \%$.

Al calcular las tasas de demanda de servicios para 2004-2008, se encontró que en 2004 el régimen contributivo presentó una tasa de utilización de servicios de $52 \%$ de su población afiliada frente a $45 \%$ por parte del régimen subsidiado, mientras que en 2006 el régimen subsidiado superó al contributivo, con tasas de $64 \%$ y $38 \%$, respectivamente - muy similares a las registradas al año siguiente. No obstante, en 2008 las tasas cayeron drásticamente, situándose en $19,7 \%$ para el régimen subsidiado y en $18 \%$ para el contributivo. En cuanto a la población no asegurada, las tasas de utilización de servicios fueron de $17 \%$ en 2004, 30\% en 2006 y $14 \%$ en 2008.

\section{DISCUSIÓN}

La OMS reporta que el $37 \%$ de los años de vida saludables (AVISA) perdi- dos a nivel mundial corresponden a los países de nivel medio de ingresos, entre los cuales se cuenta Colombia (11). La literatura sobre carga de enfermedad en este país muestra un mayor peso de las patologías crónicas, especialmente en mortalidad prematura y discapacidad, además de reducciones en la prevalencia e incidencia de patologías transmisibles $(7,12,13)$.

El análisis de morbilidad en Colombia basado en datos de los RIPS reveló una gran demanda de servicios relacionados con la atención de enfermedades transmisibles, que se debe tener en cuenta a la hora de formular y ejecutar políticas de prestación y financiación de servicios de salud. La presencia de una demanda importante de atención para patologías transmisibles no solo ha sido reportada para muestras de la población colombiana, sino también en las de otros países latinoamericanos como Bolivia, Perú y Venezuela (14-17). Al mismo tiempo, otros países de la Región muestran un proceso de transición epidemiológica más completo, con tasas de patologías crónicas más altas $(18,19)$.

Dado el alto porcentaje de población afiliada al régimen subsidiado, era de esperar que se registrara una mayor cantidad de eventos en este régimen que en los otros dos, tal como ocurrió a partir de 2006 (20). Llama la atención sin embargo la relativamente alta proporción de servicios prestados a la población no asegurada (17\% en 2004, 30\% en 2006 y $14 \%$ en 2008), dado que según las cifras oficiales no debería ser superior a $10 \%$ (21).

Los resultados del presente estudio muestran que las patologías transmisibles, perinatales, maternas y nutricionales son más frecuentes en los grupos de edad inferiores, hallazgo consistente con lo observado en otros países latinoamericanos (22). A su vez, los grupos de edad superiores, especialmente de 45 años en adelante registran una cantidad importante de eventos relacionados con patologías no transmisibles, contrariamente a lo que ocurre con las infecciones en los mayores de 60 años de edad, presentes en apenas 12,8\% de los diagnósticos. En la población de 15 a 44 años se encontró un pequeño repunte de patologías transmisibles debido a que en dicho segmento se contaron procedimientos ginecológicos (p. ej. abortos) que son de alta incidencia en este grupo de edad.

En todos los años y escenarios estudiados se observó una mayor tasa de servicios para las mujeres. Se halló, por ejemplo, un mayor porcentaje de patologías transmisibles en el género femenino que 
en el masculino, probablemente debido a la presencia de infecciones y otras patologías gineco-obstétricas. En cambio, se encontró una mayor demanda de servicios para atención de externalidades en hombres que en mujeres, especialmente en la población de entre 5 y 44 años de edad (12\% vs. 3,2\% de los diagnósticos, respectivamente). Este dato es de especial relevancia para un país como Colombia, con un conflicto armado interno de grandes dimensiones y una carga de enfermedad en este grupo de patologías 15\% superior al promedio regional (23). Aun así, la importancia de las externalidades como diagnóstico ha sido reportada para otros países de la Región que no sufren conflictos internos tan importantes, como Perú y Venezuela $(11,14,24)$.

El análisis de diagnósticos por escenario reveló que a partir de 2006 las patologías transmisibles incrementaron su importancia relativa en urgencias y decrecieron en hospitalización. Aun cuando la información disponible no permitió determinar la causa de este comportamiento, es posible que se haya debido a que la creciente cobertura del sistema de salud colombiano se haya traducido en una mayor consulta en urgencias por patologías transmisibles, reduciéndose el número de complicaciones que requieren hospitalización. Otra razón, opuesta a la anterior, puede haber sido que por problemas en los servicios de consulta externa haya aumentado la demanda en la atención de urgencias.

Se encontró además un aumento en la cantidad -y utilización- de los servicios pertenecientes al régimen contributivo durante 2004-2005, seguido de un cambio en la relación hacia un aumento en los servicios del régimen subsidiado a partir de 2006. En este sentido, las cifras de cobertura del seguro de salud colombiano informados en la literatura muestran que en 2004 el régimen subsidiado superó al contributivo porcentualmente, por lo que el aumento en la demanda de servicios sería consistente con la dinámica del esquema de seguros (8-10). Para 2008 se observó una tasa menor de eventos, lo que podría deberse a un subregistro de datos durante ese año.

En cuanto al análisis por región geográfica, se observó una preponderancia de patologías transmisibles con respecto a las no transmisibles en departamentos selváticos y costeros del país - resultados similares a los reportados en Perú (22). Estas regiones se caracterizan por menores densidades de población y escaso acceso a servicios públicos en general y sanitarios en particular. (De hecho, en 2010, la región del Caribe y la Amazonía-Orinoquía fueron los lugares del país con menor tasa de utilización de servicios de salud.)

Sin embargo, no todos los departamentos costeros o selváticos mostraron un aumento de patologías transmisibles con respecto a las no transmisibles. Tales fueron los casos, por ejemplo, de Caquetá, Chocó, Córdoba y Magdalena, departamentos que mostraron una relación transmisibles/no transmisibles promedio para el período de 0,66:1, 0,43:1, 0,47:1 y 0,39:1 respectivamente. Esta mayor prevalencia de patologías transmisibles en regiones apartadas con dificultades de acceso a servicios e infraestructura ya ha sido descrita en otros países de la Región $(16,17)$. Incluso cuando - como ocurre con la morbilidad por escenario- la causa de este comportamiento es difícil de establecer, es probable que radique en la presencia de barreras para el acceso a la atención en salud, las cuales reducirían las oportunidades de asistir a consultas en los servicios y estimularían a la población a buscar soluciones médicas alternativas (p. ej. la medicina tradicional o la consulta a farmacéuticos).

El aumento en las patologías no transmisibles es un fenómeno característico de sociedades en proceso de transición demográfica (11), las cuales se caracterizan por tener de un lado una disminución de la tasa de fecundidad y del otro una disminución de la tasa de mortalidad (12, 25-27). Sin embargo, en el caso de Colombia dicha evolución ha sido desigual, lo cual podría explicar la diferencia en la composición de las causas de morbilidad para las diferentes regiones del país. Tal dinámica demográfica se traduce en un perfil mezclado de demanda de servicios de salud para patologías transmisibles y no transmisibles, generando una carga mayor —en términos de recursos y planificaciónpara el sistema de salud, fenómeno ya observado por ejemplo en México y en otros países de la Región con niveles de ingreso medio y bajo $(11,28)$.

No se puede hablar entonces de una transición demográfica y epidemiológica completa, ya que hay una persistencia importante de patologías transmisibles.

Aun así, la migración de colombianos al extranjero (estimada en $10 \%$ de la población y que afecta en particular al grupo de edad de entre 15 y 35 años) y una potencial mejora en infraestructura, acceso a servicios públicos y atención en salud plantean para el futuro un avance en la transición demográfica y una mayor incidencia de enfermedades no transmisibles, especialmente en áreas urbanas (25).

Hay que señalar que, si bien los RIPS ofrecen información importante para el análisis de la morbilidad, la disponibilidad de datos ha venido descendiendo: en 2008 solo $33 \%$ de las administradoras de servicios de salud del país presentaron informes sobre enfermedades, frente al $45 \%$ que lo hicieron en 2005. Tal reducción podría responder a sesgos en la gestión de los datos, ya que algunos departamentos o tipos de organizaciones reportan de forma consistente, dejando regiones y tipos de afiliación sin la cobertura informativa adecuada. Afortunadamente, la información disponible corresponde a administradoras de afiliación en salud que cubren alrededor de $50 \%$ de los usuarios en el país, es decir una proporción importante de la población para la cual hay datos disponibles. En este contexto, es importante el desarrollo a futuro de un sistema integral que recoja, genere y ofrezca información completa, sólida y pertinente acerca de la actividad del sector salud.

A pesar de contar con los registros de prestación de servicios de un porcentaje importante de la población colombiana, una porción de esta continúa sin acceso a los servicios (razón por la cual la morbilidad no queda registrada en la base de datos), lo que podría representar un sesgo de información. Asimismo, se encontraron algunos problemas de calidad en las bases de datos que pudieran haber alterado en alguna medida las estimaciones relacionadas con las frecuencias diagnósticas.

\section{Conclusiones}

Aun cuando se hallaron algunos problemas de calidad menores en las bases de datos utilizadas y pese a que solo se reporta alrededor de la mitad de la información sobre morbilidad en Colombia, los RIPS constituyen una valiosa fuente informativa. En este sentido, es perentorio fortalecer y mejorar estos registros mediante el desarrollo de mecanismos de trabajo conjunto entre el gobierno y las entidades encargadas de recolectar, organizar y reportar datos sobre la mor- 
bilidad en el país. Estos esfuerzos deberían apuntar al logro de una cobertura de información que abarque a toda o por lo menos a la gran mayoría de la población colombiana.

Se ha demostrado que las enfermedades transmisibles son más frecuentes en los grupos de edad inferiores, mientras que las no transmisibles se concentran más en los grupos de edad mayores. $\mathrm{Al}$ mismo tiempo, las externalidades (p. ej. accidentes, lesiones auto-infligidas y violencia) aparecen como una causa de mor- bilidad importante que afecta con mayor fuerza a los grupos de entre 5 y 44 años de edad. Estos hallazgos deben tenerse en cuenta al momento de replantear y desarrollar políticas de salud, de modo que prevengan y protejan a los diferentes grupos de población según su menor o mayor riesgo de padecer problemas de salud determinados.

No se puede hablar de una transición epidemiológica completa en Colombia. $\mathrm{Si}$ bien han aumentado los diagnósticos de enfermedades no transmisibles -especialmente en algunas regiones del país- las infecciones siguen teniendo una presencia relevante en todos los grupos de edad y escenarios de prestación de servicios de salud, sobre todo en departamentos con deficiencias de infraestructura y acceso a los servicios. Tal situación exige revisar las políticas sanitarias para orientarlas no solo a mejorar la salud de la población sino también a reducir la brecha de servicios que existe entre las diferentes regiones del país.

\section{REFERENCIAS}

1. Organización Panamericana de la Salud. Mejoramiento de la estructura y desempeño de los sistemas nacionales de información en salud: enfoque operacional y recomendaciones estratégicas. Santiago, Chile: OPS; 2009. Disponible en: http://new.paho.org/chi/ images/PDFs/sistemas\%20nacionales $\% 20$ informaci\%F3n\%20salud_final.pdf Acceso el 7 de setiembre de 2011.

2. Aguilar Romo M, Muñoz Baeza S, De la Paz García B. La importancia de la información de la salud para la toma de decisiones. Calimed. 1998;4(2):36-7.

3. Sistema Integral de Información de la Protección Social (SISPRO). Información General del SISPRO. Bogotá, Colombia: Ministerio de la Protección Social; 2011. Disponible en: http:// www2.sispro.gov.co/Paginas/Informacion SISPRO.aspx Acceso el 7 de setiembre de 2011.

4. Ortiz Martínez RG, Díaz-Granados Sánchez KP. Análisis técnico de los Registros Individuales de Prestación de Servicios en Salud. Observatorio de Salud Pública de Santander. 2006;2(1):17-21.

5. Departamento Nacional de Estadística (DANE). Series de población 1985-2020. Bogotá, Colombia: DANE; 2011. Disponible en:http://www.dane.gov.co/daneweb_V09/ index.php?option $=$ com_content $\&$ view $=$ article\&id=238\&Itemid=121 Acceso el 7 de setiembre de 2011.

6. World Health Organization. The global burden of disease 2004, update. Geneve: OMS; 2008. Disponible en: http://www.who.int/ healthinfo/global_burden_disease/2004 report_update/en/index.html Acceso el 7 de setiembre de 2011.

7. Acosta Ramírez N, Peñaloza RE, Rodríguez García J. Carga de enfermedad Colombia 2005: Resultados alcanzados. Documento técnico ASS/1502-08. Bogotá, Colombia: Cendex-Pontificia Universidad Javeriana; 2008. Disponible en: http://www.cendex.org.co/ GPES/informes/PresentacionCarga Informe.pdf Acceso el 7 de setiembre de 2011.

8. Así Vamos en Salud. Indicadores de aseguramiento, porcentaje de población afiliada al Sistema General de la Seguridad Social en Salud. Bogotá, Colombia: Así Vamos en Salud; 2011. Disponible en: http://www. asivamosensalud.org/index.php?option= com_content\&view=article\&id=201:porcent aje-de-poblacion-afiliada-al-sgsss\&catid=59: Indicadores\&Itemid=73 Acceso el 8 de setiembre de 2011.

9. Así Vamos en Salud. Indicadores de aseguramiento, porcentaje de cobertura de afiliación al régimen contributivo. Bogotá, Colombia: Así Vamos en Salud; 2011. Disponible en: http:// www.asivamosensalud.org/index. php?option=com_content\&view=article\&id= 199:porcentaje-de-cobertura-de-afiliacion-alregimen-contributivo\&catid=59:Indicadores \&Itemid=73 Acceso el 8 de setiembre de 2011.

10. Así Vamos en Salud. Indicadores de aseguramiento, porcentaje de cobertura por afiliación al régimen subsidiado. Bogotá, Colombia: Así Vamos en Salud; 2011. Disponible en: http://www.asivamosensalud.org/index. php?option=com_content\&view $=$ article\&id $=$ 200:porcentaje-de-cobertura-por-afiliacion-alregimen-subsidiado\&catid=59:Indicadores $\&$ Itemid=73 Acceso el 8 de setiembre de 2011.

11. World Health Organization. Global health risks: mortality and burden of disease attributable to selected major risks. Geneve: WHO; 2009. Disponible en: http://www. who.int/healthinfo/global_burden_disease/ global_health_risks/en/index.html Acceso el 8 de setiembre de 2011.

12. Organización Mundial de la Salud. Estadísticas sanitarias mundiales 2011. Francia: OMS; 2011. Disponible en: http://www.who.int/ whosis/whostat/ES_WHS2011_Full.pdf Acceso el 8 de setiembre de 2011.

13. Organización Panamericana de la Salud. Análisis del perfil de salud urbana en Bogotá. Bogotá, Colombia: OPS; 2009. Disponible en: http://new.paho.org/col/ index.php?option $=$ com_docman\&task $=$ doc download\&gid=434\&Itemid $=$ Acceso el 8 de setiembre de 2011.

14. Pan American Health Organization. Health situation in the Americas, Basic Indicators 2009. Washington, D.C.: 2009. Disponible en: http://new.paho.org/hq/dmdocuments / 2009/BI_ENG_2009.pdf Acceso el 8 de setiembre de 2011.

15. Alarcón Villaverde J. Perú: El perfil epidemiológico en un contexto de demanda reprimida de servicios de salud (Documento 2). Lima, Perú: U. S. Agency for International Develop- ment; 2003. Disponible en: www.health systems2020.org/files/1620_file_Tech025s fin.pdf Acceso el 8 de setiembre de 2011.

16. Organización Panamericana de la Salud. Salud en las Américas 2007: Bolivia. Volumen II-Países. Santiago, Chile: OPS; 2007. Pp. 13247. (Publicación científica y técnica No. 622). Disponible en: http://www.paho.org/hia/ archivosvol2/paisesesp/Bolivia\%20Spanish. pdf Acceso el 8 de setiembre de 2011.

17. Organización Panamericana de la Salud. Salud en las Américas 2007: Venezuela. Volumen II-Países. Santiago, Chile: OPS; 2007. Pp. 770-84. (Publicación científica y técnica No. 622). Disponible en: http://www.paho. org/hia/archivosvol2/paisesesp/Venezuela \%20Spanish.pdf Acceso el 8 de setiembre de 2011.

18. Organización Panamericana de la Salud. Salud en las Américas 2007: Argentina. Volumen II-Países. Santiago, Chile: OPS; 2007. Pp. 38-61. (Publicación científica y técnica No. 622). Disponible en: http://www.paho.org/ hia/archivosvol2/paisesesp/Argentina\%20 Spanish.pdf Acceso el 8 de setiembre de 2011.

19. Organización Panamericana de la Salud. Salud en las Américas 2007: Chile. Volumen II-Países. Santiago, Chile: OPS; 2007. Pp. 196218. (Publicación científica y técnica No. 622). Disponible en: http://www.paho.org/hia/ archivosvol2/paisesesp/Chile\%20Spanish. pdf Acceso el 8 de setiembre de 2011.

20. Ministerio de Protección Social. Sector de la Protección Social, Informe de Actividades 2009-2010, Informe al Congreso. Bogotá, Colombia: Ministerio de la Protección Social; 2010. Disponible en: http://www.min proteccionsocial.gov.co/Documentos $\% 20$ $\mathrm{y} \% 20 \mathrm{Publicaciones} / \mathrm{INFORME} \% 20 \mathrm{AL}$ $\%$ 20CONGRESO $\% 202009-2010 \% 20 Y \% 20$ CUATRENIO\%202006-2010.pdf Acceso el 8 de setiembre de 2011.

21. Así Vamos en Salud. Indicadores de aseguramiento, porcentaje de población no asegurada. Bogotá, Colombia: Así Vamos en Salud; 2011. Disponible en: http://www.asivamosen salud.org/index.php?option=com_content\& view $=$ article \&id $=129:$ Porcentaje $\% 20 \mathrm{de} \% 20$ poblaci\%C3\%B3n\%20no\%20asegurada\& catid=59:Indicadores\&Itemid=73 Acceso el 8 de setiembre de 2011. 
22. Instituto Nacional de Estadística e Informática del Perú. Encuesta demográfica y de salud familiar 2010. Lima, Perú: INEI; 2011. Disponible en: http://proyectos.inei.gob.pe/ endes/endes2010/resultados/index.html Acceso el 8 de setiembre de 2011.

23. World Health Organization. Country Health Profile: Colombia. Geneve: WHO; 2010. Disponible en: http://www.who.int/countries/ $\mathrm{col} / \mathrm{en} /$ Acceso el 8 de setiembre de 2011.

24. World Health Organization. Country Health Profile: Venezuela. Geneve: WHO; 2010. Disponible en: http://www.who.int/countries/ ven/en/ Acceso el 8 de setiembre de 2011.
25. Grajales IC, Cardona D. La segunda transición demográfica y el nivel de desarrollo de los departamentos de Colombia. Universidad de Antioquia: Revista Facultad Nacional de Salud Pública. 2005;28(3):209-20.

26. Carmona-Fonseca J. Cambios demográficos y epidemiológicos en Colombia durante el siglo XX. Biomédica. 2005;25(4):464-80.

27. Organización Panamericana de la Salud. Salud en las Américas 2007: Colombia. Volumen II-Países. Washington, D.C.: OPS; 2007. Pp. 220-41. (Publicación científica y técnica No. 622). Disponible en: http://www.paho. org/hia/archivosvol2/paisesesp/Colombia
\%20Spanish.pdf Acceso el 8 de setiembre de 2011.

28. Arredondo A, Zúñiga A, Parada I. Health care costs and financial consequences of epidemiological changes in chronic diseases in Latin America: evidence from Mexico. Public Health. 2005;119(8):711-20.

Manuscrito recibido el 23 de marzo de 2011. Aceptado para publicación, tras revisión, el 1 de agosto de 2011.

ABSTRACT Objective. To characterize morbidity in the Colombian population by diagnosis, sex, age, region, and health coverage system, and evaluate the availability and quality of information on diseases in the country. A descriptive, cross-sectional study to

Data availability and morbidity profile in Colombia analyze morbidity treated in outpatient visits, hospitalization, and emergencies in Colombia during the 2004-2008 five-year period.

Methods. Based on Colombia's personal health records (Registro Individual de Prestación de Servicios-RIPS), diagnoses were classified according to World Health Organization cause groups. Each cause group for services utilization was differentiated by geographical region, sex, and type of affiliation to the health system.

Results. Communicable disease diagnoses were more concentrated in younger age groups, while noncommunicable diseases were more frequent in older age groups. External causes (accidents, self-inflicted injuries, and violence) were a major cause of morbidity and more frequently affected the population aged 5-44 years. Communicable diseases were more prevalent in females $(39.98 \%)$ than in males $(28 \%)$, while males were more affected by external causes than females $(18.5 \%$ and $7.3 \%$, respectively).

Conclusions. Although diagnoses of noncommunicable diseases have increased in Colombia, infections continue to have an important presence in all age groups and health services delivery settings. This situation requires a review of health policies, not only to orient them toward improving the health of the population, but also toward bridging the services gap that exists among the country's different regions. Furthermore, although RIPS are a valuable source of health data, they need to be strengthened with a view to achieving information coverage for all or at least the vast majority of Colombians.

Key words Morbidity; information systems; diseases registries; medical records; Colombia. 